\title{
Anisotropic Solutions in the 5D Space-Time-Mass Theory*
}

\author{
W. L. Roque ${ }^{\dagger}$ and W. M. Seiler \\ Institut für Algorithmen und Kognitive Systeme \\ Universität Karlsruhe \\ D-7500 Karlsruhe 1, Germany
}

\begin{abstract}
In this paper we present anisotropic families of cosmological solutions in the 5-dimensional Space-Time-Mass Theory of Gravity. In particular, the 5D analogue of the Kasner solution of General Relativity is obtained. Some comments are given to the solutions.
\end{abstract}

\section{Introduction}

It has been a long standing desire that all physical theories have both properties: coordinate-invariance and scale-invariance. The theory of General Relativity has the former but not the latter one. Many attempts have so far been made to accomplish these properties in an unique theory of gravity. In this direction many authors have suggested alternative theories to the General Relativity of Einstein. The main ones were proposed by Dirac in his Large Number Hypothesis Theory [1], followed by Hoyle and Narlikar [2], Canuto et al. [3] and others.

${ }^{*}$ Supported by CNPq - Brazil.

†Permanent address: Universidade de Brasília, Departamento de Matemática, 70910 Brasília DF, Brazil. E-mail: roq@ilncc.bitnet. 
These alternative theories share the property of varying the gravitational constant $G$ and/or the rest mass of objects with time in a scale of the order of the age of the universe $\left(10^{10} \mathrm{yr}\right)$. Although they have shed some light on the problem of scale-invariance, they all have been discarded based on observational grounds. In addition, these theories always appeal to a two metric approach, one describes the gravitational physics and the other refers to atomic physics, which is neither mathematically nor physically very clear.

In a set of recent papers $[4,5]$ an alternative theory of gravity has been proposed, where the Newtonian gravitational constant $G$ is not chosen to be time-varying, but rather the mass is varying. The physical arguments for this choice are well established in the papers [5, 6]. Although the theory proposed is not a pure scale-invariant theory of gravity, as has been pointed out by Grøn and Soleng [7] (see the comments in [8]), it is a scale-free gravity theory, as the rest mass is variable and so does not define a scale as in GR.

In this new theory the space-time is no longer described by a $4 \mathrm{D}$ manifold but by a 5D space-time-mass (STM) Riemannian manifold where the mass plays the role of a fifth coordinate given by $x^{4}=\frac{G m}{c^{2}}$. The Einstein theory is recovered when the velocity $\frac{G}{c^{2}} \frac{d m}{d t}=0$. In other words, when the mass is constant. In this regard, the 4D space-time theory of Einstein can be thought of as embedded in the 5D STM theory.

This paper is concerned with possible exact anisotropic solutions for an extension of the GR spatially homogeneous and anisotropic Bianchi type I cosmological model to the 5D STM theory. In section 2 we give the mathematical description of the space-time-mass theory; section 3 is concerned with the field equations for a Bianchi type I metric and separation of variables. Section 4 treats the power-law ansatz and families of solutions are given. In section 5 some comments and conclusions are addressed to the paper.

\section{The STM field equations}

The theory developed in $[4,5]$ assumes that the space-time-mass can be described by a 5-dimensional manifold with the line element

$$
d s^{2}=g_{i j} d x^{i} d x^{j}, \quad i, j=0, \ldots, 4 .,
$$

where $x^{0}=c t, x^{1,2,3}=$ space coordinates and $x^{4}=G \mathrm{~m} / \mathrm{c}^{2}$ (mass coordinate). Here the mass coordinate $x^{4}$, which has dimension of length, is measured with 
respect to an arbitrary origin, in much the same way as the time and space coordinates $t, x^{1,2,3}$. For later purpose, we shall label the mass coordinate as $x^{4} \equiv w$.

As the metric signature of the STM theory is not well established, it can be either $+1(-1)$ or $+3(-3)$ according to the sign of the fifth coordinate ([7], [8]), for the time being, we shall take it unspecified by chosing it to be $(-1,+1,+1,+1, \epsilon)$, where the parameter can be $\epsilon= \pm 1$.

The field equations of the STM theory is just the 5D analogue of the General Relativity field equations, namely:

$$
G_{i j}=R_{i j}-\frac{1}{2} R g_{i j}=\frac{8 \pi G}{c^{4}} T_{i j}
$$

In the field equations above, $T_{i j}$ is the energy-momentum tensor ${ }^{1}$.

Exact solutions of the STM field equations have been found for the onebody problem (see [5]), leading to the Schwarzschild solution in the limit of constant mass, and for vacuum homogeneous and isotropic metrics by Chatterjee [9], Fukui [10], and later extensively studied by Ponce de Leon [11]. More recently, a new homogeneous and isotropic solution appeared in $[12]^{2}$, which represents a singularity free expanding universe with the compactification of the fifth dimension as the universe evolves. Cosmological solutions for radiation and dust were found by Grøn [13] considering a 5D extension of FRW metric.

\section{Bianchi type I}

In a recent paper, Ponce de Leon [11] has presented a serie of solutions for a homogeneous and spatially isotropic metric in the 5D STM theory.

We shall consider now the 5D extension of the spatially homogeneous and anisotropic Bianchi type I space-time metric, given by

$$
d s^{2}=-d t^{2}+R_{1}^{2} d x^{2}+R_{2}^{2} d y^{2}+R_{3}^{2} d z^{2}+\epsilon R_{4}^{2} d w^{2},
$$

where $R_{i}, i=1, \ldots, 4$ are functions of $t$ and $w$, only.

\footnotetext{
${ }^{1}$ There are some claims that the STM field equations might well be $G_{i j}=0$, but no definite results.

${ }^{2}$ We thank the referee for pointing out this reference.
} 
The sign of the mass coordinate has been left free as it is a controversial matter $[5,7,8]$. However, it can be easily chosen by fixing the value of the parameter $\epsilon$. In fact, the sign can be, at least in principle, determined experimentally by means of the conservation equations [5].

The vacuum field equations for the metric given by 3 are easily obtained with the aid of the computer algebra system REDUCE [14]:

$$
\begin{aligned}
& \frac{\ddot{R}_{1}}{R_{1}}+\frac{\ddot{R}_{2}}{R_{2}}+\frac{\ddot{R}_{3}}{R_{3}}+\frac{\ddot{R}_{4}}{R_{4}}=0, \\
& \frac{\dot{R}_{1}^{\prime}}{R_{1}}+\frac{\dot{R}_{2}^{\prime}}{R_{2}}+\frac{\dot{R}_{3}^{\prime}}{R_{3}}-\frac{\dot{R}_{4}}{R_{4}}\left(\frac{R_{1}^{\prime}}{R_{1}}+\frac{R_{2}^{\prime}}{R_{2}}+\frac{R_{3}^{\prime}}{R_{3}}\right)=0, \\
& R_{1}^{2}\left\{\frac{\ddot{R}_{1}}{R_{1}}+\frac{\dot{R}_{1}}{R_{1}}\left(\frac{\dot{R}_{2}}{R_{2}}+\frac{\dot{R}_{3}}{R_{3}}+\frac{\dot{R}_{4}}{R_{4}}\right)\right. \\
& \left.-\frac{1}{\epsilon} \frac{1}{R_{4}^{2}}\left[\frac{R_{1}^{\prime \prime}}{R_{1}}+\frac{R_{1}^{\prime}}{R_{1}}\left(\frac{R_{2}^{\prime}}{R_{2}}+\frac{R_{3}^{\prime}}{R_{3}}-\frac{R_{4}^{\prime}}{R_{4}}\right)\right]\right\}=0, \\
& \epsilon R_{4}^{2}\left[\frac{\ddot{R}_{4}}{R_{4}}+\frac{\dot{R}_{4}}{R_{4}}\left(\frac{\dot{R}_{1}}{R_{1}}+\frac{\dot{R}_{2}}{R_{2}}+\frac{\dot{R}_{3}}{R_{3}}\right)\right]-\frac{R_{1}^{\prime \prime}}{R_{1}}-\frac{R_{2}^{\prime \prime}}{R_{2}}-\frac{R_{3}^{\prime \prime}}{R_{3}} \\
& +\frac{R_{4}^{\prime}}{R_{4}}\left(\frac{R_{1}^{\prime}}{R_{1}}+\frac{R_{2}^{\prime}}{R_{2}}+\frac{R_{3}^{\prime}}{R_{3}}\right)=0,
\end{aligned}
$$

where the dot means derivative with respect to $t$ and the prime means derivative with respect to $w$. The field equation 6 is cyclic in the space coordinates $(1,2,3)$.

\subsection{Separation of variables}

The field equations 4 to 7 are not trivial to be fully solved. Therefore, to proceed further let us assume that the metric coefficients can be written as a product of functions with separate variables. In other words, that

$$
R_{i}(t, w)=S_{i}(t) Q_{i}(w), \quad i=1, \ldots, 4 .
$$

Substituting now the functions 8 into the vacuum field equations, we get, after some manipulations

$$
\frac{\ddot{S}_{1}}{S_{1}}+\frac{\ddot{S}_{2}}{S_{2}}+\frac{\ddot{S}_{3}}{S_{3}}+\frac{\ddot{S}_{4}}{S_{4}}=0
$$




$$
\begin{gathered}
S_{4}^{2}\left[\frac{\ddot{S}_{1}}{S_{1}}+\frac{\dot{S}_{1}}{S_{1}}\left(\frac{\dot{S}_{2}}{S_{2}}+\frac{\dot{S}_{3}}{S_{3}}+\frac{\dot{S}_{4}}{S_{4}}\right)\right]= \\
+\frac{1}{\epsilon} \frac{1}{Q_{4}^{2}}\left[\frac{Q_{1}^{\prime \prime}}{Q_{1}}+\frac{Q_{1}^{\prime}}{Q_{1}}\left(\frac{Q_{2}^{\prime}}{Q_{2}}+\frac{Q_{3}^{\prime}}{Q_{3}}-\frac{Q_{4}^{\prime}}{Q_{4}}\right)\right]=\text { const. } \\
S_{4}^{2}\left[\frac{\ddot{S}_{4}}{S_{4}}+\frac{\dot{S}_{4}}{S_{4}}\left(\frac{\dot{S}_{1}}{S_{1}}+\frac{\dot{S}_{2}}{S_{2}}+\frac{\dot{S}_{3}}{S_{3}}\right)\right]= \\
+\frac{1}{\epsilon} \frac{1}{Q_{4}^{2}}\left[\frac{Q_{1}^{\prime \prime}}{Q_{1}}+\frac{Q_{2}^{\prime \prime}}{Q_{2}}+\frac{Q_{3}^{\prime \prime}}{Q_{3}}-\frac{Q_{4}^{\prime}}{Q_{4}}\left(\frac{Q_{1}^{\prime}}{Q_{1}}+\frac{Q_{2}^{\prime}}{Q_{2}}+\frac{Q_{3}^{\prime}}{Q_{3}}\right)\right]=\text { const. } \\
\left(\frac{\dot{S}_{1}}{S_{1}}-\frac{\dot{S}_{4}}{S_{4}}\right) \frac{Q_{1}^{\prime}}{Q_{1}}+\left(\frac{\dot{S}_{2}}{S_{2}}-\frac{\dot{S}_{4}}{S_{4}}\right) \frac{Q_{2}^{\prime}}{Q_{2}}+\left(\frac{\dot{S}_{3}}{S_{3}}-\frac{\dot{S}_{4}}{S_{4}}\right) \frac{Q_{3}^{\prime}}{Q_{3}}=0 .
\end{gathered}
$$

The equation 10 is cyclic in the space coordinates $(1,2,3)$. Notice that only the equation 12 cannot be decoupled.

General solution of these equations seems not trivial to be found, too. However, further simplifications can be achieved, if we consider an assumption that solves equation 12 . This can be obtained, when we consider either

(i) $Q_{1}^{\prime} / Q_{1}=Q_{2}^{\prime} / Q_{2}=Q_{3}^{\prime} / Q_{3}=0$, and $Q_{4} \neq 0$, or

(ii) an isotropic expansion rate.

\subsubsection{Case (i)}

Case (i), after some manipulations of the equations, shows that the expansion rates $\dot{S}_{i} / S_{i}$ differ by a constant factor from each other. In other words,

$$
\frac{\dot{S}_{i} / S_{i}}{\dot{S}_{j} / S_{j}}=\text { const., } i \neq j=1, \ldots, 4,
$$

and, in addition,

$$
\mathcal{S}^{4} \equiv S_{1} S_{2} S_{3} S_{4}=c t+d, \quad \dot{c}=\dot{d}=0 .
$$

Taking into account these two relations and equation 9, one can show that for an appropriate choice of initial time we get

$$
\begin{gathered}
S_{i}=c_{i} t^{a_{i}}, \quad \dot{c}_{i}=0, \\
\sum_{i=1}^{4} a_{i}=\sum_{i=1}^{4} a_{i}^{2}=1,
\end{gathered}
$$


which represents a 5D extension of the Kasner solutions of General Relativity. The average rate of expansion becomes

$$
\frac{\dot{\mathcal{S}}}{\mathcal{S}} \propto \frac{1}{t},
$$

which shows a time singularity as $t \rightarrow 0$.

This result is a generalization of the solution $2(W=$ const., $N=$ const. $)$ found in Ponce de Leon's paper.

\subsubsection{Case(ii)}

On the other hand, if we consider now that the expansion rates are the same in every direction (isotropy), we get, using equation 9, that

$$
S_{i}=p t+q, \dot{p}=\dot{q}=0, i=1, \ldots, 4 .
$$

For an appropriate choise of initial time, we get also that

$$
\frac{\dot{\mathcal{S}}}{\mathcal{S}} \propto \frac{1}{t},
$$

which is singular at $t \rightarrow 0$.

If we consider the particular case of $p=0^{3}$ in equation 18 , which leads to vanishing separation constants, and define $\gamma_{i}=Q_{i}^{\prime} / Q_{i}, i=1, \ldots, 4$, we obtain from equations 9 to 12 that

$$
\gamma_{1}=-\frac{\gamma_{2} \gamma_{3}}{\gamma_{2}+\gamma_{3}}
$$

The particular solution of 20 for $\gamma_{i}=$ const. $, i=2,3,4$ is given by

$$
\begin{aligned}
& Q_{1}=q_{1} e^{-\frac{\gamma_{2} \gamma_{3}}{\gamma_{2}+\gamma_{3}} w}, Q_{2}=q_{2} e^{\gamma_{2} w}, \\
& Q_{3}=q_{3} e^{\gamma_{3} w}, \quad Q_{4}=q_{4} e^{\gamma_{4} w}, \quad q_{i}=\text { const. },
\end{aligned}
$$

which for $w \rightarrow 0$ becomes 5 D Minkowski space-time-mass and for $w \rightarrow \infty$, $Q_{1} \rightarrow 0$ while $Q_{2,3,4} \rightarrow \infty$.

These solutions extend the solution 1 found in Ponce de Leon's paper (his case for $S=$ const. and $K=$ const.).

\footnotetext{
${ }^{3}$ Notice that $p=0$ leads to static space-time-mass solutions.
} 


\section{Power-law solutions}

To be able to carry on the search for new exact anisotropic solutions, let us n ow assume the power-law ansatz for the functions $S_{i}(t)$ and $Q_{i}(w)$ :

$$
S_{i}(t)=t^{a_{i}} \quad, \quad Q_{i}(w)=w^{b_{i}},
$$

where $a_{i}$ and $b_{i}, i=1, \ldots, 4$ are constants.

Substituting into the field equations (9 to 12), after some manipulations, we get

$$
\begin{aligned}
a_{1}^{2}+a_{2}^{2}+a_{3}^{2}+a_{4}^{2}-a_{1}-a_{2}-a_{3}-a_{4} & =0, \\
b_{1}\left(a_{1}-a_{4}\right)+b_{2}\left(a_{2}-a_{4}\right)+b_{3}\left(a_{3}-a_{4}\right) & =0, \\
a_{i}\left(a_{1}+a_{2}+a_{3}+a_{4}-1\right) t^{2\left(a_{4}-1\right)}+ & \\
\frac{1}{\epsilon} b_{i}\left(1+b_{4}-b_{1}-b_{2}-b_{3}\right) w^{-2\left(1+b_{4}\right)} & =0, \\
a_{4}\left(a_{1}+a_{2}+a_{3}+a_{4}-1\right) t^{2\left(a_{4}-1\right)}+ & \\
\frac{1}{\epsilon}\left[\left(b_{1}+b_{2}+b_{3}\right)\left(b_{4}+1\right)-\left(b_{1}^{2}+b_{2}^{2}+b_{3}^{2}\right)\right] w^{-2\left(1+b_{4}\right)} & =0,
\end{aligned}
$$

where in equation $25 i=1,2,3$.

The complete solution ${ }^{4}$ of the above set of equations is found when we consider the two cases:

(a) $a_{4}=-b_{4}=1$,

(b) the coefficients of $t$ and $w$ vanish simultaneously.

\subsection{Case (a)}

Here we have two distinct solutions depending upon the sign of the parameter $\epsilon$.

\footnotetext{
${ }^{4}$ The solution $a_{i}=b_{i}=0$ has been discarded as it is mathematically and physically trivial.
} 


\subsubsection{Solution for $\epsilon=-1$}

This leads to the simple solution $a_{i}=b_{i}=0, i=1,2,3$. The line element for this solution is given by

$$
d s^{2}=-d t^{2}+d x^{2}+d y^{2}+d z^{2}-\frac{t^{2}}{w^{2}} d w^{2}
$$

which represents a flat space-time-mass.

\subsubsection{Solution for $\epsilon=+1$}

For $\epsilon=+1$, we obtain a 2-parameter family of solutions given by:

Family 1: $a_{4}=-b_{4}=1$

$$
\begin{aligned}
b_{1 \pm}=a_{1 \pm} & =\frac{1}{2}(1 \pm \sqrt{1-4 \alpha(\alpha-1)-4 \beta(\beta-1)}), \\
b_{2}=a_{2} & =\alpha \\
b_{3}=a_{3} & =\beta
\end{aligned}
$$

where the solutions must have the values of the parameters $\alpha$ and $\beta$ lying on or inside the circumference $(\alpha-1 / 2)^{2}+(\beta-1 / 2)^{2}=3 / 4$.

The solutions with $\alpha=\beta=0, \alpha=0$ and $\beta=1, \alpha=1$ and $\beta=0$, or $\alpha=\beta=1$ are all flat space-time-mass solutions.

The Kretschmann scalar $R^{i j k l} R_{i j k l}$ for this family vanishes.

A non-flat solution is found for $\alpha=0, \beta=1 / 2$. Its line element is given by

$$
d s^{2}=-d t^{2}+(t w)^{1 \pm \sqrt{2}} d x^{2}+d y^{2}+t w d z^{2}+\frac{t^{2}}{w^{2}} d w^{2}
$$

which has the determinant given by

$$
g=-t^{4}(t w)^{ \pm \sqrt{2}}
$$

For the minus sign solution it is singular at $w=0$ and becomes indetermined when $t \rightarrow \infty$. 


\subsection{Case (b)}

The case (b) establishes the set of equations

$$
\begin{aligned}
a_{1}^{2}+a_{2}^{2}+a_{3}^{2}+a_{4}^{2}-a_{1}-a_{2}-a_{3}-a_{4}=0, \\
b_{1}\left(a_{1}-a_{4}\right)+b_{2}\left(a_{2}-a_{4}\right)+b_{3}\left(a_{3}-a_{4}\right)=0, \\
a_{i}\left(a_{1}+a_{2}+a_{3}+a_{4}-1\right)=0, \\
b_{j}\left(b_{1}+b_{2}+b_{3}-b_{4}-1\right)=0, \\
\left(b_{1}+b_{2}+b_{3}\right)\left(b_{4}+1\right)-\left(b_{1}^{2}+b_{2}^{2}+b_{3}^{2}\right)=0,
\end{aligned}
$$

where in equation $35 i=1, \ldots, 4$ and in equation $36 j=1,2,3$.

All solutions of these equations are found, when the following conditions are independently satisfied

(b1) $a_{i}=0, i=1, \ldots 4$, or

(b2) not all the $a_{i}$ vanish.

The condition (b1) above implies that all solutions under it represent static space-time-mass solutions. Notice that the sign of $\epsilon$ plays no role in the system.

\subsubsection{Solution for (b1)}

Under condition (b1), the system of equations 33 to 37 reduces to

$$
\begin{aligned}
b_{j}\left(b_{1}+b_{2}+b_{3}-b_{4}-1\right) & =0, \\
\left(b_{1}+b_{2}+b_{3}\right)\left(b_{4}+1\right)-\left(b_{1}^{2}+b_{2}^{2}+b_{3}^{2}\right) & =0 .
\end{aligned}
$$

The general solution of the above system is found when we consider the following sub-conditions:

i. $b_{j}=0, j=1,2,3$,

ii. not all $b_{j}$ vanishes. 


\section{Case (b1i)}

If all $b_{j}=0$, we have a 1 -parameter family of solutions given by

Family 2: $a_{i}=0, i=1, \ldots, 4, \quad b_{j}=0, j=1,2,3$

$$
b_{4}=\lambda
$$

However, this is not an interesting family, as it corresponds to flat spacetime-mass.

\section{Case (b1ii)}

To cover all solutions in (b1ii), we should consider the sub-cases ${ }^{5} b_{2}+b_{3} \neq$ 0 and $b_{2}+b_{3}=0$, which lead to a 2-parameter and 1-parameter families, respectively.

The Kretschmann scalar for for (b1ii) is given by

$$
R^{i j k l} R_{i j k l}=\frac{16 \lambda^{2} \mu^{2}\left(\lambda^{2}+\lambda \mu+\mu^{2}\right)}{(\lambda+\mu)^{2} w^{\frac{4\left(\lambda^{2}+\lambda \mu+\mu^{2}\right)}{\lambda+\mu}}},
$$

which shows a singularity at $w=0$, unless when $\lambda=\mu=0$ as the spacetime-mass is flat (see family 4 below).

Family 3: $b_{2}+b_{3} \neq 0$

$$
\begin{aligned}
& b_{1}=-\frac{\lambda \mu}{\lambda+\mu}, b_{2}=\lambda, b_{3}=\mu, \\
& b_{4}=\frac{\lambda^{2}+(\lambda+\mu)(\mu-1)}{\lambda+\mu} .
\end{aligned}
$$

The determinant of the metric for this case is given by

$$
g=-\epsilon w^{2\left(1+2 b_{4}\right)},
$$

\footnotetext{
${ }^{5}$ The choice of $b_{2}$ and $b_{3}$ in what follows is completely arbitrary, any other two in $\left(b_{1}, b_{2}, b_{3}\right)$ would well suit for the choice.
} 
which is singular for $w \rightarrow 0$ when $b_{4}<-1 / 2$.

The solutions with either $\lambda=0$ or $\mu=0$ are all flat space-time-mass solutions. A particular non-flat space-time-mass solution is found for $\lambda=$ $\mu=1$. The line element becomes

$$
d s^{2}=-d t^{2}+\frac{1}{w} d x^{2}+w^{2} d y^{2}+w^{2} d z^{2}+\epsilon w d w^{2}
$$

Family 4: $b_{2}+b_{3}=0$

The solution of the system 38 to 39 gives a 1-parameter family,

$$
\begin{aligned}
& b_{1}=\mu, \mu \neq 0 \\
& b_{2}=b_{3}=0 \\
& b_{4}=\mu-1
\end{aligned}
$$

These solutions are all flat space-time-mass.

\subsubsection{Solution for (b2)}

Under condition (b2) the system of equations 33 to 37 becomes

$$
\begin{aligned}
a_{1}+a_{2}+a_{3}+a_{4}=a_{1}^{2}+a_{2}^{2}+a_{3}^{2}+a_{4}^{2} & =1, \\
b_{1}\left(a_{1}-a_{4}\right)+b_{2}\left(a_{2}-a_{4}\right)+b_{3}\left(a_{3}-a_{4}\right) & =0, \\
b_{j}\left(b_{1}+b_{2}+b_{3}-b_{4}-1\right) & =0, \\
\left(b_{1}+b_{2}+b_{3}\right)\left(b_{4}+1\right)-\left(b_{1}^{2}+b_{2}^{2}+b_{3}^{2}\right) & =0 .
\end{aligned}
$$

To solve this system we should consider the same conditions (i) and (ii) as for case (b1).

The equations 46 show that we cannot have exactly two of the $a_{i}, i=$ $1, \ldots, 4$ vanishing.

\section{Case (b2i)}

The case (b2i) leads to a 3-parameter family of solutions given by

Family $5: b_{j}=0, j=1,2,3$ 


$$
\begin{aligned}
b_{4} & =\lambda \\
\sum_{i=1}^{4} a_{i} & =\sum_{i=1}^{4} a_{i}^{2}=1 .
\end{aligned}
$$

If we choose $a_{3}$ and $a_{4}$ as parameters, we can write the equations above as follows:

$$
\begin{aligned}
& b_{4}=\lambda, \\
& a_{1}=\frac{1}{2}\left(1-\alpha-\nu+\sqrt{1+2 \alpha-2 \alpha \nu+2 \nu-3 \alpha^{2}-3 \nu^{2}}\right), \\
& a_{2}=\frac{1}{2}\left(1-\alpha-\nu-\sqrt{1+2 \alpha-2 \alpha \nu+2 \nu-3 \alpha^{2}-3 \nu^{2}}\right), \\
& a_{3}=\alpha, \quad a_{4}=\nu .
\end{aligned}
$$

These solutions must have the values of the parameters $\alpha$ and $\nu$ lying on or inside the shifted and rotated ellipse given by $\frac{4}{3}(\alpha+\nu-1 / 2)^{2}+\frac{2}{3}(\alpha-\nu)^{2}=1$.

All the solutions with $\alpha=\nu=0$ correspond to a flat space-time-mass, otherwise they are 5D Kasner-like solutions.

As the values of $a_{i}$ 's are parameter-wise independent of $\lambda$, the 5D analogue of Kasner solutions of General Relativity are obtained when we set the value of $\lambda=0$. In particular, Kasner solutions of GR are obtained at every slice of constant mass $(d w=0)$ as long as the GR conditions $\sum_{i}^{3} a_{i}=\sum_{i}^{3} a_{i}^{2}=1$ are satisfied. The easiest ones are those with $\nu=0$.

The Kretschmann scalar for family 5 is given by

$$
\begin{aligned}
R^{i j k l} R_{i j k l}= & -\frac{8}{t^{4}}\left\{2 \alpha^{2}(\alpha-1)+2 \nu^{2}(\nu-1)\right. \\
& +\alpha \nu[\alpha(\alpha+1)+\nu(\nu+1)+\alpha \nu-2]\}
\end{aligned}
$$

which exhibits a singularity for $t \rightarrow 0$.

From equations 46 and 48 the determinant of the metric for this family becomes

$$
g=-\epsilon t^{2} w^{2 b_{4}}
$$

Thus, for $b_{4}<0, g$ has a (mass) singularity as $w \rightarrow 0$ unless when $t \rightarrow \infty$, where it becomes indetermined.

A 1-parameter family of solutions which for every hypersurface of constant mass represents a non-flat space-like singularity free family and which is not 
a Kasner ${ }^{6}$ solution of General Relativity is given by $\alpha=0$ and $\nu=-1 / 3$. This leads to the line element

$$
d s^{2}=-d t^{2}+t^{4 / 3} d x^{2}+t^{4 / 3} d y^{2}+d z^{2}+\epsilon \frac{1}{t^{2 / 3}} \frac{1}{w^{2 \lambda}} d w^{2} .
$$

Wesson's solutions in [6], eq. 6, and in [15], eq. 12, correspond to the particular solutions of Family 5 with $\epsilon=-1, \lambda=0$ and $a_{1}=a_{2}=a_{3}=$ $1 / 2, a_{4}=-1 / 2$ and $a_{1}=a_{2}=a_{3}=0, a_{4}=1$, respectively.

In order to take account of the variation of the rest mass of a test particles, let us consider the geodesic equations,

$$
\frac{d^{2} x^{i}}{d s^{2}}+,{ }_{j k}^{i} \frac{d x^{j}}{d s} \frac{d x^{k}}{d s}=0
$$

for the 5D Kasner-like family of solutions $\left(b_{4}=0\right)$.

After some manipulations with the geodesic equations, we get

$$
\begin{aligned}
\frac{d w}{d s} & =\frac{t_{w}}{t^{2 a_{4}}}, \\
\frac{d t}{d s} & =\sqrt{\left(\frac{t_{x}}{t^{a_{1}}}\right)^{2}+\left(\frac{t_{y}}{t^{a_{2}}}\right)^{2}+\left(\frac{t_{z}}{t^{a_{3}}}\right)^{2}+\epsilon\left(\frac{t_{w}}{t^{a_{4}}}\right)^{2}+t_{k}^{2}}, \\
\frac{d w}{d t} & =\frac{t_{w}}{t^{2 a_{4}}\left(\frac{d t}{d s}\right)},
\end{aligned}
$$

where $t_{x}, t_{y}, t_{z}, t_{w}, t_{k}$ are constants.

A naive interpretation of eq. 54 is that for $t_{w}>0$ the rest mass increases along $s$ and decreases for $t_{w}<0$. However, for $a_{4}<0$ the variation becomes very small for earlier times, while for $a_{4}>0$ it approaches a constant value for later times. Therefore, the universe models in the former case may not need to have an initial singularity, while in the latter case they have an initial singularity and approaches the GR behaviour at later times.

Notice that the eq. 55, and consequently 56, do depend explicitly on the metric signature through the value of the parameter $\epsilon$.

\section{Case (b2ii)}

In the case (b2ii), the solutions of the equations 46 to 49 are split into

\footnotetext{
${ }^{6}$ Kasner solutions in GR cannot have just one of the power coefficients vanishing.
} 
two classes $^{7}$ as follows.

Case (b2ii): solutions for $b_{2}+b_{3} \neq 0$

Solving equation 49 taking into account equation 48 we obtain that

$$
\begin{aligned}
& b_{1}=-\frac{\lambda \mu}{\lambda+\mu}, \quad b_{2}=\lambda, \quad b_{3}=\mu, \\
& b_{4}=\frac{\lambda^{2}+(\lambda+\mu)(\mu-1)}{\lambda+\mu}, \\
& a_{1}=\frac{(1-2 \nu)(\lambda+\mu)^{2}-2 \lambda \mu \nu+\left(\lambda^{2}-\mu^{2}\right) \sqrt{1+4 \nu-8 \nu^{2}}}{2\left(\lambda^{2}+\lambda \mu+\mu^{2}\right)}, \\
& a_{2}=\frac{2 \lambda \nu(\lambda+\mu)-(2 \nu-1) \mu^{2}+\mu(2 \lambda+\mu) \sqrt{1+4 \nu-8 \nu^{2}}}{2\left(\lambda^{2}+\lambda \mu+\mu^{2}\right)}, \\
& a_{3}=\frac{2 \mu \nu(\lambda+\mu)-(2 \nu-1) \lambda^{2}-\lambda(\lambda+2 \mu) \sqrt{1+4 \nu-8 \nu^{2}}}{2\left(\lambda^{2}+\lambda \mu+\mu^{2}\right)}, \\
& a_{4}=\nu .
\end{aligned}
$$

where $\lambda, \mu$ and $\nu$ are free parameters. Therefore, we have a 3 -parameter family of solutions.

All these solutions can be covered by taking three 2-parameters families of solutions, which are given below by Family 6,7 and 8 , except when $a_{4} \neq$ $0, b_{2} \neq-b_{3} \neq 0$, where they are specifically determined by the eqs. 57 to 62 .

Family 6: $b_{2}=\lambda=0,(\mu \neq 0)$

$$
\begin{aligned}
& b_{1}=0, \quad b_{3}=\mu, \quad b_{4}=\mu-1, \\
& a_{1}=\frac{1}{2}\left(1-2 \nu-\sqrt{1+4 \nu-8 \nu^{2}}\right), \\
& a_{2}=\frac{1}{2}\left(1-2 \nu+\sqrt{1+4 \nu-8 \nu^{2}}\right), \\
& a_{3}=a_{4}=\nu .
\end{aligned}
$$

Notice that the solutions are parameter-wise completely decoupled. The physical solutions must have the values of $\nu$ constrained to the range $\nu \in$

\footnotetext{
${ }^{7}$ Here too, the choice of $b_{2}$ and $b_{3}$ in what follows is completely arbitrary, any other two in $\left(b_{1}, b_{2}, b_{3}\right)$ would well suit for the choice.
} 
$\left[\frac{1-\sqrt{3}}{4}, \frac{1+\sqrt{3}}{4}\right]$. In particular, solutions with $\nu=0$ are all flat space-time-mass solutions.

The Kretschmann scalar for this family is given by

$$
R^{i j k l} R_{i j k l}=-\frac{24}{t^{4}} \nu^{2}\left(\nu^{2}+\nu-2\right),
$$

which shows that we have only a singularity at $t=0$, unless for $\nu=0$, which is flat (the values $\nu=-1 \pm \sqrt{3}$ are out of the physical range).

A 5D Kasner-like solution is found for $\mu=1, \nu=2 / 3$, where the line element becomes:

$$
d s^{2}=-d t^{2}+t^{-2 / 3} d x^{2}+d y^{2}+t^{4 / 3} w^{2} d z^{2}+\epsilon t^{4 / 3} d w^{2} .
$$

Family 7: $b_{3}=\mu=0,(\lambda \neq 0)$

This family can be written out from Family 6 just by relabeling $\mu\left(b_{3}\right) \rightarrow$ $\lambda\left(b_{2}\right)$.

Family 8: $a_{4}=\nu=0,(\lambda \neq-\mu \neq 0)$

The values of $b_{1}, b_{2}, b_{3}$ and $b_{4}$ are as in 57 to 58 and

$$
\begin{aligned}
& a_{1}=\frac{\lambda(\lambda+\mu)}{\mu^{2}+\lambda(\lambda+\mu)}, \\
& a_{2}=\frac{\mu(\lambda+\mu)}{\lambda^{2}+\mu(\lambda+\mu)}, \\
& a_{3}=-\frac{\lambda \mu}{\lambda^{2}+\lambda \mu+\mu^{2}} .
\end{aligned}
$$

For $m=$ const. hypersurfaces, all solutions correspond to a Kasner solution of $\mathrm{G} R$.

A particular 5D Kasner-like solution is found when $\mu=\lambda=1$, which gives the line element

$$
d s^{2}=-d t^{2}+\frac{t^{4 / 3}}{w} d x^{2}+t^{4 / 3} w^{2} d y^{2}+\frac{w^{2}}{t^{2 / 3}} d z^{2}+\epsilon w d w^{2} .
$$

The Kretschmann scalar for this metric is given by

$$
R^{i j k l} R_{i j k l}=\frac{12}{w^{6}}-\frac{8}{3 \epsilon t^{2} w^{3}}+\frac{64}{27 t^{4}},
$$


which is singular for both $t \rightarrow 0$ and $w \rightarrow 0$.

Case (b2ii): solutions for $b_{2}+b_{3}=0$

In this case, from 46 to 49 , we have only one independent 2-parameter family of solutions given by:

Family 9: $b_{1} \neq 0$

$$
\begin{aligned}
& b_{1}=\mu, \quad b_{2}=b_{3}=0, \\
& b_{4}=\mu-1, \\
& a_{1}=a_{4}=\nu, \\
& a_{2}=\frac{1}{2}\left(1-2 \nu+\sqrt{1+4 \nu-8 \nu^{2}}\right), \\
& a_{3}=\frac{1}{2}\left(1-2 \nu-\sqrt{1+4 \nu-8 \nu^{2}}\right) .
\end{aligned}
$$

Notice that this family, although independent, can be obtained from Family 6 just by relabeling $a_{3} \leftrightarrow a_{1}$ and $b_{3} \leftrightarrow b_{1}$. Therefore, we get here similar physical conditions and the same Kretschmann scalar as in Family 6.

If we take $\mu=1$ and $\nu=1 / 2$, we get a particular solution with line element given by

$$
d s^{2}=-d t^{2}+t w^{2} d x^{2}+t d y^{2}+\frac{1}{t} d z^{2}+\epsilon t d w^{2},
$$

which shows a time singularity for $t \rightarrow 0$ and a mass singularity for $w \rightarrow 0$.

\section{Conclusion}

In this paper we have considered an extension to the spatially homogeneous and anisotropic Bianchi type I cosmological model in the scale-free 5D SpaceTime-Mass Theory of Gravity developed in [4, 5]. Exact 5D Kasner-like families of solutions have been found and the Kasner solutions of GR were obtained as a particular case.

The extended Bianchi type I space-time metric was considered to be only function of the time $t$ and mass $w$ coordinates. As the signature of the space-

time-mass line element is not yet well defined, we left in all computations the 
sign of the fifth coordinate free through the parameter $\epsilon= \pm 1$. The vacuum field equations for the STM theory are given in 4 to 7 .

To simplify the field equations, the metric coefficients were assumed to be the product of two functions in separate variables. The field equations shown to be not fully decoupled. Two particular cases were investigated: Firstly, when $Q_{i}^{\prime} / Q_{i}=0, i=1,2,3$ and $Q_{4}$ is left free (case (i), section 3 ). As a consequence of that, the time-dependent functions are just power-law functions obeying a 5D analogue of the Kasner solution in General Relativity (see equation 16) and the expansion rates in each direction are proportional to each other (anisotropy). Secondly, the isotropic case $\left(\dot{S}_{i} / S_{i}=\dot{S}_{j} / S_{j}, i=\right.$ $1, \ldots, 4$, case (ii), section 3 ) was considered which shows that the metric time dependent functions must be linear in $t$. In both cases the average rate of expansion $\dot{\mathcal{S}} / \mathcal{S}$ is proportional to $1 / t$. In particular, case (ii) also allows for static solutions as a by-product (see eqs. 22).

To be able to further simplify, we have chosen the power-law ansatz (see 22 ) for the metric functions. The vacuum field equations have been completely solved leading to 9 different families of solutions.

Family 1 , with $\epsilon=1$, is a 2 -parameter solution with $a_{i}=b_{i}, i=1,2,3$ and $a_{4}=-b_{4}=1$. The line element of a non-flat solution of this family is given in 31 , which has the Kretschmann scalar vanishing.

The Families 2, 3 and 4 are all families of static solutions, as all the $a_{i}, i=1, \ldots, 4$ vanish. Families 2 and 4 have no physical interest, as they are flat space-time-mass. Family 3 is a 2-parameters family. A particular non-flat solution of Family 3 is given by the line elements 45 .

Family 5 is a 3 -parameter family of solutions with $b_{j}=0, j=1,2,3$. The 5D analogue of Kasner solutions of GR are obtained when one of the parameters, $\lambda$ for this case, is set equal zero. In particular, the Kasner solutions of GR are regained at $d w=0$ hypersurfaces as long as the GR conditions $\sum_{i}^{3} a_{i}=\sum_{i}^{3} a_{i}^{2}=1$ are satisfied. A 1-parameter sub-family of Family 5 where the hypersurfaces of constant mass $(d w=0)$ are not Kasner solutions of GR is shown by the line element 52 .

The solutions found by Wesson in [6] (his eq. 6) and in [15] (his eq. 12), are just two particular cases $\left(\epsilon=-1, b_{4}=0, a_{1}=a_{2}=a_{3}=-a_{4}=1 / 2\right.$ and $a_{1}=a_{2}=a_{3}=0, a_{4}=1$, respectively) of Family 5 .

According to the geodesic equations, the rest mass of a test particle in a 5D Kasner-like expanding universe (see eq. 54) may either approaches a finite constant value at very early times and increases afterwards, when 
$a_{4}<0$, or have an infinite initial value and becomes constant at later times, when $a_{4}>0$.

Thus, the universe models with $a_{4}>0$ seem to be more realistic universe models as presently observations indicate an initial Big Bang singularity and no variation in the rest mass of particles (it might had been stronger at the very early universe and very small at the present time) in contrast to the universe models with $a_{4}<0$, which may not have the initial singularity but on the other hand they would provide a stronger variation in the rest mass of particles which could perhaps be easier detected. These universe models exhibit a natural compactification of the mass coordinate as they expand (this seems to be the case presented in [12]).

A deeper investigation on the variation of rest mass of particles based in the universe models presented in this paper is in progress and will appear elsewhere.

Families 6,7 and 8 are all 2-parameter families of solutions satisfying the conditions (b2ii) and $b_{2}+b_{3} \neq 0$, while Family 9 is a 2-paramter family of solutions satisfying the conditions (b2ii) and $b_{2}+b_{3}=0$. The solutions of (b2ii) with $a_{4} \neq 0$ and $b_{2} \neq-b_{3} \neq 0$ are given directly by the eqs. 57 to 62 .

To have an idea of the kinematical [16] behaviour of these solutions, let us consider a congruence of small test particles with 5 -velocity $u_{i}$ such that $u^{i} u_{i}=-1$ and $u_{i}=-\delta_{i}^{0}$, then the 4 -volume expansion $\Theta$ and the shear magnitude $\sigma^{2}$ (anisotropy) of this congruence are given, respectively, by

$$
\begin{aligned}
\Theta & =\frac{\sum a_{i}}{t}, \\
\sigma^{2} & =\frac{\sum_{i<j}^{4}\left(a_{i}-a_{j}\right)^{2}}{8 t^{2}} .
\end{aligned}
$$

From equations 75 and 76 we see that the space-time-mass for all families but Families 2, 3 and 4 (they are static) are slowing down their expansion and isotropizing in a similar way as the Bianchi I universe model of General Relativity. An expanding shear free solution (isotropic expansion) is found in Family 1 when $a_{i}=1$.

Notice that the sign of $\epsilon$ plays no role for the expansion or anisotropy and that both are independent of the mass coordinate ${ }^{8}$.

\footnotetext{
${ }^{8}$ This is somehow expected due to the choice of comoving test particles.
} 
Although we have started off the paper taking the fifth coordinate as being mass, mathematically speaking the solutions found here are independent of the physical nature of the fifth coordinate. Therefore, they are new (as far as the authors are concerned) exact power-law solutions in a 5D pseudoRiemannian manifold. However, as the role of the mass in the STM theory is not yet fully understood, neither in its geometrical contents nor in its physical realm, we think that new exact solutions together with astrophysical and cosmological considerations should bring some additional informations, and as such, they need to be further investigated. It is our hope that the anisotropic solutions presented here can be used as the starting point to investigate the behaviour of the rest mass of particles in more realistic universe models.

The STM theory of gravity has many points that need a better understanding, particularly in the cosmological level where real departures from General Relativity may be expected. Nevertheless, at the present stage of development, the astrophysical data available do not discard the possibility of a slow variation of the rest mass of particles on a cosmological scale. In fact, an experiment to investigate very small departures on the rest mass of bodies (order of 1 part in $10^{10}$ per year) is under consideration [17] for some time and it is expected to give a definite result in the near future. This would certainly establish or not the STM as a viable theory of gravity.

\section{Acknowledgment}

W.L. Roque thanks Prof. J. Calmet for the warm hospitality at the IAKS, I. A. Tjandra for many technical computational assistance and the Conselho Nacional de Desenvolvimento Científico e Tecnológico - CNPq for the financial support. 


\section{References}

[1] Dirac, P.A.M. (1973). Proc. Roy. Soc. London, A333, 403.

[2] Hoyle, F. and Narlikar, J.V. (1974). Action at a Distance in Physics and Cosmology, Freeman, San Francisco, USA.

[3] Canuto, V., Adams, P.J., Hsieh, S.-H. and Tsiang, E. (1977). Phys. Rev., D33, 1643.

[4] Wesson, P.S. (1983). Astron. Astrophys., 119, 145.

[5] Wesson, P.S. (1984). Gen. Rel. Grav., 16, 193.

[6] Wesson, P.S. (1986). Astron. Astrophys., 166, 1.

[7] Grøn, Ø. and Soleng, H.H. (1988). Gen. Rel. Grav., 20, 1115.

[8] Wesson, P.S. (1990). Gen. Rel. Grav., 22, 707.

[9] Chatterjee, S. (1986). Gen. Rel. Grav., 18, 1073.

[10] Fukui, T. (1987). Gen. Rel. Grav., 19, 43.

[11] Ponce de Leon, J. (1988). Gen. Rel. Grav., 20, 593.

[12] Chi, L. K. (1990). Gen. Rel. Grav., 22, 1347.

[13] Grøn, Ø. (1988). Astron. Astrophys., 193, 1.

[14] Hearn, A.C. (1987). REDUCE User's Manual, version 3.3, The Rand Corporation, Santa Monica, CA.

MacCallum, M.A.H. and Wright, F. Algebraic Computing with REDUCE, Lecture Notes from the First Brazilian School on Computer Algebra, vol. I, eds. Rebouças, M. J. and Roque, W. L. (Forthcoming book Oxford University Press, 1991).

[15] Wesson, P.S. (1988). Astron. Astrophys., 189, 4.

[16] Ellis, G.F.R. (1971). In General Relativity and Cosmology, Proceedings of the XLVII Enrico Fermi Summer School, ed. R. K. Sachs (Academic Press). 
[17] Ritter, R.C., Gilles, G.T., Rood, R.T and Beams, J.W. (1978). Nature, 271, 228. 